\title{
REVIEW OF SURFACE RELAXATION AND RECONSTRUCTION PHENOMENA
}

\author{
P. ZIELIŃSKI
}

Institute of Nuclear Physics, Radzikowskiego 152, 31-342 Kraków, Poland

The basic definitions concerning structure of surface are given. The principal experimental methods of the observation of surfaces are reviewed. A possibly heuristic explanation is given of principal theoretical concepts underlying the present understanding of the surface phenomena. The best known and/or understood examples of the surface relaxation, surface reconstruction and roughening are reviewed.

PACS numbers: $68.35 .-\mathrm{p}, 68.35 . \mathrm{Bs}, 68.35 . \mathrm{Md}, 68.35 . \mathrm{Rh}$

\section{Introduction: basic definitions}

Any crystalline solid can be considered as a series of mutually parallel crystallographic lattice planes, arranged in space in a periodic way. Every two such planes separated by a multiple of the spatial period have identical structure in the bulk of the crystal. Close to the surface, however, the spatial arrangement of the lattice planes, their crystallographic structure as well as the dynamics of the constituent atoms may differ from the corresponding features in the bulk. The very special and, in fact, rather rare case of no variation either of the spatial arrangement or of the structure of the lattice planes close to the surface will be called ideal unreconstructed surface.

Surface relaxation is a rigid shift of one or more entire lattice planes located near to the surface with respect to their positions characteristic of the bulk. One distinguishes parallel and perpendicular relaxation according to whether the shift is parallel or perpendicular to the surface. The name relaxation comes from the fact that if we cleave an initially ideal unreconstructed surface the structure of the system will then relax under the unbalanced forces produced by the removal of a part of the crystal.

Whenever the structure of one or more topmost lattice planes differs from the structure of the corresponding lattice planes in the bulk one speaks of a reconstructed surface. Reconstructed surfaces show a two-dimensional periodicity, which may be identical or different from the periodicity of the corresponding lattice plane in the bulk. One characterizes the reconstruction by specifying the reconstructed two-dimensional unit cell in terms of the two-dimensional lattice parameters of the unreconstructed surface. For example the notation

$$
c(\sqrt{2} \times 3 \sqrt{2}) R 45^{\circ}(001)
$$


means that the period of the reconstructed surface in the direction (10) is multiplied by $\sqrt{2}$ and the period in the direction (01) is multiplied by $3 \sqrt{2}$ with respect to the periods (10) and (01) of the unreconstructed lattice plane. The letter $c$ indicates a centered structure of the reconstructed surface, whereas a letter $p$ in the same position would correspond to a primitive structure. The letter $R$ followed by an angle defines the rotation of the reconstructed unit cell with respect to the original unreconstructed two-dimensional lattice. The last three figures in the parentheses are the Miller indices which define the orientation of the surface with respect to the three-dimensional crystal lattice. Variations of temperature or of the coverage of adsorbed atoms may provoke transformations from one reconstructed structure to another. These transformations are a kind of structural phase transitions in two dimensions.

There also exist crystalline surfaces where the atoms from the topmost lattice plane do not form any periodic 2D structure. One then speaks of disordered (flat) surface.

All the above described surfaces are well localized and have finite thickness. They are called smooth. There exist, however, surfaces which do not show these properties. Their atoms stick out from a reference level on very different heights so that one cannot any more speak of a finite thickness. Such surfaces are called rough.

Considering the crystal-with-surface as a 2D array of atomic columns labelled by indices $i j$ and having the heights $z_{i j}$ one defines the rough surface as the one for which the mean squared height difference

$$
G_{i j i^{\prime} j^{\prime}}=\left\langle\left(z_{i j}-z_{i^{\prime} j^{\prime}}\right)^{2}\right\rangle \text {, }
$$

where the \langle\rangle means averaging over the whole surface, goes to infinity when the distance $\left|r_{i j}-r_{i^{\prime} j^{\prime}}\right| \rightarrow \infty$. For smooth surfaces the mean squared height difference remains finite even for infinitely distant sites. A smooth surface may roughen under variation of temperature or of the coverage of adsorbed atoms. The corresponding transformation is a very peculiar phase transition called roughening phase transition which occurs only in two dimensions.

The high-index or vicinal surfaces, i.e. the surfaces making a small angle with a low-index plane, are usually stepped. This means that if one tries to cleave a surface of this orientation, the system of steps will arise so as to make the surface consist of small portions of low-index, usually more stable surfaces. Vicinal surfaces also undergo roughening transition.

The roughening phase transition and the transition to the disordered structure should be distinguished from the surface melting. In this latter case not only does the topmost layer lose its periodicity, but also the atoms become mobile and leave their well defined lattice sites, so that their state is rather liquid than solid. In all the other mentioned cases the surface is a kind of a two-dimensional crystalline or amorphous solid deposited on a three-dimensional solid of the bulk crystal.

In this review we shall present the most known and studied examples of the surface relaxation, reconstruction and roughening along with the theoretical models aimed at the explanation of the experimental findings in terms of microscopic atomic interactions. A brief review of experimental techniques of observations are given in Sec. 2. Sections 3 and 4 present current theoretical concepts concerning 
structure of surfaces. Examples of surface relaxation, reconstruction and roughening are given in Secs. 5, 6 and 7 respectively.

\section{Experimental techniques for observation of crystal surfaces}

\subsection{Macroscopic techniques}

Smooth and rough surfaces of a given material can be distinguished by insight into the equilibrium shape of the crystal. Whereas the smooth surfaces form flat planar "facets", the rough surfaces are rounded. When a crystal in equilibrium is heated through the roughening temperature of one of its surfaces, the corresponding facet disappears. In this way the roughening temperatures and some information on the step interaction have been obtained for surfaces of metallic crystals: $\mathrm{Ag}, \mathrm{Cu}$ and $\mathrm{Pb}$ [1]. A variant of this method is the observation of "negative crystals", i.e. vapour bubbles included within a crystal. Roughening of some molecular crystals: biphenyl, naphtalene and tetrabromomethane has been so detected [2]. A disadvantage of this technique is that the time needed to reach the equilibrium shape of a crystal is long and increases rapidly with the crystal size. Consequently, the observed shapes of crystals usually do not correspond to the equilibrium state, but are rather a result of a specific process of the crystal growth. The non-equilibrium growth shapes are, nevertheless, also useful in the study of rough surfaces. This comes from the fact that the rate of growth is much higher for rough surfaces than for the smooth ones. A comparison of the growth of differently oriented surfaces of $\mathrm{Au}, \mathrm{In}$ and $\mathrm{Pb}$ has allowed for the detection of roughening transitions in these metals [3].

\subsection{Scattering experiments}

The majority of the known cases of surface relaxation and surface reconstruction have been obtained by scattering methods with different radiations. Two difficulties have to be overcome when applying a radiation to study the structure of surfaces. Firstly, the radiation cannot penetrate too deeply into the crystal so as to convey the information from the surface layer only. Secondly, the scattering from the surface being weak compared with the scattering from the bulk, one has to use intense sources of radiation. In numerous X-ray experiments the shallow penetration has been achieved by a glancing incidence of less than $1^{\circ}$ within the angle of total external reflection. The required intensity of the radiation is available from synchrotron facilities. This technique allowed for the determination of very large number of known cases of the surface reconstruction and surface relaxation. The usefulness of the neutron scattering at grazing angles is restricted to surfaces of sufficiently large area because of the low scattering intensity.

Another possibility of reduction of the penetration depth is offered by the use of a radiation strongly interacting with the matter. Many important results have been obtained by means of the low-energy electron diffraction (LEED) mostly applied with normal incidence of $25-500 \mathrm{eV}$. In the reflection high-energy electron diffraction (RHEED) the electrons with grazing incidence have the energy of 5-15 keV. Many reconstructed structures have been solved by these techniques in spite of the difficulty in interpretation of the spectra related to a rather high 
contribution of multiple scattering produced by electron beams. Inelastic electron scattering (EELS - electron energy loss spectroscopy) is used to measure surface dispersion relations.

Still lower energies $(\leq 0.1 \mathrm{eV})$ are used in the experiments with the He atoms scattering. This method, or generally thermal energy atom scattering (TEAS), are particularly useful in the study of dynamics of the surfaces [4]. As the probe radiation one uses also ion beams. The low energy $(\approx 1 \mathrm{keV})$ ion scattering spectroscopy (ISS) and the high-energy $(\approx 1 \mathrm{MeV})$ Rutherford backscattering spectroscopy (RBS) are widely used to determine surface structures.

In all the scattering experiments the structures of surfaces are deduced from the positions of the diffraction or Bragg peaks. The latter ones are always superimposed on the diffuse background. The presence of more or less correlated defects and fluctuations related to the appearance of superstructures gives a special feature to the diffuse scattering. The study of the shape of peaks allows one to deduce the form of the correlation functions which are predicted by theory.

\subsection{Atomic scale microscopy}

Scanning tunnelling microscopy (STM) and atomic force microscopy (AFM) are new powerful tools which permit direct observations of surfaces. They are particularly well adapted to studies of surfaces which do not evolve too fast with time. A spectacular success of the STM was the resolution of the intriguing for long time structure of the $(7 \times 7)(111) \mathrm{Si}$. A great number of reports appear every month on the STM, AFM and related techniques applied to clean surface as well as to the adsorbed layers as complicated as liquid crystals deposited on solid surfaces. The power of these techniques resides in their possibility of visualization of electronic structure and chemical bonds of the surface atoms.

\section{Surface energetics and phase transitions}

\section{1. $0 \mathrm{~K}$ siruclures}

Relaxation and reconstruction phenomena are, generally, a result of different coordination of the bulk and the surface atoms. Different coordination influences the distribution of the electrons surrounding the atoms. Consequently, the equilibrium structure of a separate lattice plane may be very different from the structure of the bulk lattice planes. The observed structure of the crystal-with-surface results, therefore, from a competition between the in-plane forces and the tendency to keep the registry with the subsurface layers.

To model a given structure of surface one has to consider so called surface energetics, i.e. find a correct form of the energy including the electronic degrees of freedom. The desired structure should then correspond to the minimum of the total energy of the system. The simplest model of ionic crystal involves point ions only. More advanced models treat the ions as consisting of positive cores and negative electronic shells linked to the cores by phenomenological harmonic forces. This is called shell model. Additionally to the Coulomb forces one can also consider repulsive and dispersion forces, e.g. in the Lennard-Jones form. The latter forces are particularly important in molecular crystals. 
The energetics of metallic and semiconductor surfaces is more complicated because of the existence of the conduction and valence electrons. Sophisticated schemes of calculation have been developed to cope with reconstructed unit cells involving hundreds of surface atoms: ab initio methods [5], embedded atom model [6], glue model [7] etc.

\subsection{Surface phase transilions}

The above described total energy calculations give in principle the ground state or the structure in $0 \mathrm{~K}$. They are, therefore, insufficient in modelling temperature-induced changes of the reconstruction. In a non-zero temperature $T$ the equilibrium state is given by the minimum of the free energy $F$ rather than of the total energy $E$ :

$$
F=E-T S,
$$

where $S$ is the entropy. This minimum reflects a compromise of the tendency of the system to minimize the total energy and maximize the entropy. The rigorous expression for the free energy involves summation over all the accessible states of the system (see Sec. 4). Taking into account the complications of the surface energetics the minimization of the exact free energy is usually unfeasible. Various simplified approaches are, therefore, used.

The simplest approach consists in selecting the apparently relevant degrees of freedom and constructing the free energy in the form of a polynomial function of the selected variables. The polynomial must be invariant under all the symmetry operations of the most symmetric out of all the conceivable structures of the considered system. The coefficients of this free energy are phenomenological functions of temperature. A stable structure or phase corresponds to the minimum of this Landau free energy. The second derivatives of the free energy define the inverse of susceptibilities conjugated with the selected degrees of freedom. In particular, if the relevant degrees of freedom are atomic displacements (displacive phase transitions), the eigenvalues of the matrix of second derivatives are squared phonon frequencies. In this approach a phase transition corresponds to a shift of the minimum between two points in the space of the selected degrees of freedom. Such a transition can be continuous, i.e. to be described by an infinitesimal shift of the minimum provided that these points represent structures of different symmetry. In such cases one of the eigenvalues, or squared phonon frequencies, tends to zero at the transition point. The corresponding mode is called soft mode. Equivalently, the corresponding susceptibility diverges. Contrary to the displacive phase transitions, the order-disorder phase transitions involve probabilities of occupancy rather than atomic displacements as the relevant degrees of freedom. Numerous conclusions can be derived by only considering the symmetry of the phases. The review articles [8] summarize such conclusions for the phase transitions on surfaces.

Another phenomenological approach to phase transitions is to neglect the complication of the surface energetics and replace it by very schematic interactions involving, e.g. the energies of the most important thermally excited surface defects. This approach requires a hypothesis about the ground state of the surface, but in return, offers a possibility of calculating the exact expression for the free energy 
at least in the vicinity of the phase transition points. Some models of this type are discussed in Sec. 4.

Fast computers allow for replacing the statistical methods by a direct solution of the equations of motion of a number of atoms with assumed interactions. Then the statistical averages are replaced by configurational (i.e. over the sample) or time averages. This method, called molecular dynamics, has been used to model the behaviour of some surfaces [9].

\section{Statistical mechanics of surfaces}

In the statistical mechanics the measurable quantities are expressed by average values calculated with the use of the Boltzmann factor of the probability distribution. The probability $P(\Psi)$ of the state $\Psi$ of the system then is related to the energy $H(\Psi)$ of the state

$$
P(\Psi)=\exp \left[-H(\Psi) / k_{\mathrm{B}} T\right],
$$

where $k_{\mathrm{B}}$ is the Boltzmann constant and $T$ is the temperature.

In practice, all the interesting averages can be evaluated from the partition function $Z$ :

$$
Z=\sum_{\Psi} P(\Psi)
$$

The sum in the last formula extends over all the states accessible to the system. The free energy of the system is

$$
F=-k_{\mathrm{B}} T \ln (Z) \text {. }
$$

The averaging being complicated in itself, one usually looks for a possibly simple potential so as to make the calculations tractable. Only in some special cases analytical expressions for the partition function can be obtained.

\subsection{The continuum approach}

The simplest model of the crystal surface reduces the material to a continuous medium. The state of the system then is defined by the height $z(x, y)$ over every point $(x, y)$ of a plane chosen for reference. A reasonable assumption is that the energy $H(\{z(x, y)\})$ of the surface is proportional to its area $A$ :

$$
\begin{aligned}
& H(\{z(x, y)\})=\gamma A, \\
& A=\int_{A_{0}} \mathrm{~d} x \mathrm{~d} y(1+\nabla z)^{2}=A_{0}+\frac{1}{2} \int_{A_{0}} \mathrm{~d} x \mathrm{~d} y(\nabla z)^{2},
\end{aligned}
$$

where $A_{0}$ is the area of the corresponding portion of the plane $(x, y)$. The simplicity of the model resides in the quadratic form of the energy (Eq. (7)) which makes the probability distribution (Eq. (3)) Gaussian. The average values then can be calculated relatively easily, e.g. the mean square difference turns out to be

$$
G_{i j i^{\prime} j^{\prime}} \approx \frac{k_{\mathrm{B}} T}{\pi \gamma} \ln \left(\frac{\left|r_{i j}-r_{i^{\prime} j^{\prime} \mid}\right|}{a}\right),
$$

where $a$ is an arbitrary length of the order of the lattice constant. 
The logarithmic function of Eq. (8) being an infinitely increasing function when $\left|r_{i j}-r_{i{ }^{\prime} j^{\prime}}\right| \rightarrow \infty$, the surface described by this model is rough at all temperatures. This model is therefore insufficient in the description of the low-index crystal surfaces which are known to be smooth below a certain temperature. The smoothness of the surface can be achieved in this type of model by adding to the energy a term

$$
V[1-\cos (2 \pi z / a)],
$$

which pins the surface at the levels of $z$ corresponding to the multiples of the lattice constant $a$ if the temperature is low enough. Then, of course, the probability density (Eq. (3)) loses its Gaussian character and the calculations become more complex. They show that the system undergoes a Kosterlitz-Thouless phase transition discussed below.

\subsection{Atomistic models: Kosterlitz-Thouless phase transition}

In more realistic discrete models the state of the system is given by a $2 \mathrm{D}$ array of atomic columns of varying heights $z_{i j}$. The variable $z$ also takes on discrete values only. In case where $z_{i j}$ is a single valued function of its indices i.e. any "overhangs" are forbidden, the model is called solid-on-solid (SOS). To give the corresponding probability distribution a Gaussian form one can define the energy as follows:

$$
H=\gamma A_{0}+\frac{\gamma}{2} \cdot \sum_{i j} \sum_{\left(i^{\prime} j^{\prime}\right)_{i j}}\left(z_{i j}-z_{\left(i^{\prime} j^{\prime}\right\rangle_{i j}}\right)^{2},
$$

where $i j$ runs over all the sites of the $2 \mathrm{D}$ array and $\left\langle i^{\prime} j^{\prime}\right\rangle_{i j}$ over the nearest neighbours of the site $i j$. In this version the model is called discrete Gaussian SOS (DGSOS). The partition function of this model turns out to be

$$
Z=Z_{0} \sum_{\left\{g_{i j}\right\}=-\infty}^{\infty} \exp \left[-\frac{1}{2 k_{\mathrm{B}} T^{\prime}} \sum_{i j i^{\prime} j^{\prime}} q_{i j} q_{i^{\prime} j^{\prime}} U\left(i j, i^{\prime} j^{\prime}\right)\right],
$$

with

$$
U\left(i j, i^{\prime} j^{\prime}\right)=-\ln \left(\frac{\left|\boldsymbol{R}_{i j}-\boldsymbol{R}_{i^{\prime} j^{\prime}}\right|}{a}\right)
$$

and

$$
T^{\prime}=\frac{\gamma}{2 \pi T}
$$

The sign $\sum^{\prime}$ means that the integers $q_{i j}$ fulfil the condition $\sum_{i j} q_{i j}=0$. The factor $Z_{0}$ in the formula (11) is the non-singular partition function calculated with the variables $z_{i j}$ treated as continuous functions of the discrete argument. Looking at the formula (11) one finds out that this partition function is analogous to the partition function of a system of charges $q_{i j}$ interacting by a logarithmic potential (Eq. (12)) in the temperature $T^{\prime}$ given by Eq. (13). This system is the 2D Coulomb gas model which is known to undergo a very peculiar phase transition called Kosterlitz-Thouless (KT) phase transition. At low "temperatures" $T$ ", or equivalently at high temperatures $T$ the charges $q_{i j}$ are mostly paired in dipoles 
whereas in high "temperatures" $T^{\prime}$ or in the low temperatures $T$ the dipoles dissociate to form a gas of free charges. The dipolar phase of the 2D Coulomb gas corresponds to the rough phase of the surface and the free-charge phase of the Coulomb gas corresponds to the smooth phase of the surface. The temperature $T$ of the roughening is $T=T_{\mathrm{R}}=2 \gamma / \pi k_{\mathrm{B}}$. The mean squared height difference is divergent

$$
G_{i j i^{\prime} j^{\prime}} \propto \ln \left(\frac{\mid \boldsymbol{R}_{i j}-\boldsymbol{R}_{i^{\prime} j^{\prime} \mid}}{a}\right)
$$

for $T>T_{\mathrm{R}}$ and convergent

$$
G_{i j i^{\prime} j^{\prime}} \propto \frac{\exp \left(-\left|\boldsymbol{R}_{i j}-\boldsymbol{R}_{i^{\prime} j^{\prime}}\right| / b\right)}{\left|\boldsymbol{R}_{i j}-\boldsymbol{R}_{i^{\prime} j^{\prime} \mid}\right|}
$$

for $T<T_{\mathrm{R}}$. The quantity " $b$ " is a finite correlation length. The behaviour of the thermodynamical quantities near to the roughening transition can be deduced from the singular part of the free energy

$$
F \propto \exp \left(- \text { const }\left|T-T_{\mathrm{R}}\right|^{-1 / 2}\right) .
$$

Because the free energy has all the derivatives continuous at $T_{R}$, no anomaly is predicted in the specific heat. A large maximum should, in turn, occur at some lower temperature. All the critical exponents are infinite in the KT transition. It has to be stressed that the KT phase transition can only occur in two dimensions [10].

Various modifications of the SOS model are conceivable and used to account for the observed effects. These models are known under the name of restricted SOS models (RSOS). The most radical restriction allows the surface atoms to occupy only two levels, $N$ and $N+1$ so that $z_{i j}=N$ or $z_{i j}=N+1$. Such an array of atoms having two accessible states is the 2D Ising model which has been solved analytically by Onsager. It shows a phase transition from the disordered flat surface to different reconstructed arrangements depending on the values of the interaction parameters. When the range of interactions takes in first neighbours only, the reconstructed phase is either unreconstructed or "ferromagnetic" where all the atoms are on the same level or shows $(\sqrt{2} \times \sqrt{2}) R 45^{\circ}$ reconstruction in which all the nearest neighbours have different levels. The phase transition in the 2D Ising model shows $\lambda$ shape singularities in the specific heat and in the susceptibility described by well established critical exponents.

Another restriction has been proposed by den Nijs [11]. In this RSOS model the neighbouring columns can differ in height either by 1 or by 2 . The energy then reads

$$
H=\kappa \sum_{i j i^{\prime} j^{\prime}} \delta\left(\left|z_{i j}-z_{i^{\prime} j^{\prime}}\right|-1\right)+\lambda \sum_{i j^{\prime} j^{\prime}} \delta\left(\left|z_{i j}-z_{i^{\prime} j^{\prime} \mid}\right|-2\right),
$$

where $\delta$ stands for the Kronecker symbol. Depending on the values of the parameters $\kappa$ and $\lambda$ the surface described by this RSOS shows a variety of phases in different temperatures. In sufficiently high temperature the surface is rough. There is also a disordered flat phase as well as the "ferromagnetic" and "antiferromagnetic" ordered phases. Among the phase transitions one can find the 
KT roughening transition, 2D Ising transition between the disordered and reconstructed flat phase and still a different transition "preroughening" phase transition between disordered flat and ordered flat surface.

To describe the (001) surface of bcc and fcc metals one uses the body centered SOS (BCSOS) due to van Beijeren [12]. In this model the two-dimensional array of the columns of atoms consists of two sublattices shifted vertically with respect to each other by the half of the lattice constant. Any two nearest neighbouring columns form an ascending chain or ladder of the nearest neighbouring atoms. The assumption of van Beijeren is that none of such ladders can be broken by a vacancy except for its ending at the surface. This assumption, which is in fact analogous to the SOS condition of no overhang, allows the model to be reduced to the known and exactly solvable "six-vertex" model used in the theory of the ice disorder. Although the expression for the partition function is lengthy, it is known analytically for all temperatures. The surface described by the BCSOS model undergoes the KT type phase transition.

\section{Relaxation of unreconstructed surfaces}

\subsection{Ionic crystals}

The low-index surfaces of ionic crystals are usually unreconstructed and their relaxation is weak. The analysis of the dispersion relations of the surface phonons on the (001) surfaces of the rock salt crystals [13] indicated a very small variation of the energy parameters caused by the cleavage. De Wette et al. [14] modelled these surfaces in total energy calculations with the use of the shell model. The model parameters have been calculated from the bulk properties. The results show that the surface atoms of higher polarizability tend to slightly sick out of the surface. The distance of the two first lattice planes contracts for all the investigated crystals by a few percent.

There exist two different (001) surfaces in $\mathrm{SrTiO}_{3}$ depending whether the topmost lattice plane is composed of $\mathrm{SrO}$ (surface I) or $\mathrm{TiO}_{2}$ (surface II). Both of them show a slight perpendicular relaxation detected by a LEED experiment [15]. The change of the first interlayer distance is $-10( \pm 2) \%$ for the surface I and $+2( \pm 2) \%$ for the surface II. The shell-model total-energy calculations [16] have shown that the electronic clouds of the $\mathrm{Ti}^{4+}$ and $\mathrm{O}^{2-}$ in the surface II overlap more than in the bulk. The same model indicated that some surface phonons soften above-to the ferrodistortive phase transition which means that a surface reconstruction should precede the bulk phase transition.

Much less is known of the surfaces of orientationally disordered ionic crystals. Zieliński and Michel [17] have predicted the surface relaxation of the (001) KCN using the interaction parameters known from the bulk properties. By approaching the structural order-disorder phase transition the relaxation should grow in value and penetrate deeper into the crystal.

\subsection{Metallic crystals}

The experimental evidence of relaxation concerns mostly metal surfaces. The fcc metals show usually multilayer perpendicular relaxation. The relaxation 
is stronger for less packed surfaces. The first layer relaxation is almost always contraction although exceptions are also known: (001)Rh and (001)Pd [18]. An alternation of sign of the relaxation of the subsurface layers is very common [19]. This effect is explained within the electrostatic models treating the ions and the conduction electrons with different degree of approximation. Contrary to this, the (0001) surfaces of some hcp metals (Be, $\mathrm{Mg}$ ) show a dilatation of the first interlayer spacing [20]. This effect is due to the formation of quasi-bonds which absorb the conduction electrons within the topmost layer.

\section{Surface reconstruction}

\subsection{Metal surfaces}

The surfaces (001) of the bcc transition metals W, Mo and Cr reconstruct below room temperature. The reconstruction of the $\mathrm{W}$ surface is the in-plane $(\sqrt{2} \times \sqrt{2}) R 45^{\circ}$ so that the surface atoms form zigzag chains [21]. The phase transition from the high-temperature unreconstructed phase is generally of the displacive type and is driven by a soft mode at the border of the two-dimensional Brillouin zone [4]. The energetics of the (001)W surface has been modelled with various level of phenomenology. Fu et al. [22] in their total energy calculations found a strong coupling between the displacements of the atoms and surface electronic states, which end up in the relative stability of the reconstructed structure. Later Fasolino and Tosatti [23] constructed a model where the electronic degrees of freedom were incorporated in the effective atom interactions. This allowed them to select the displacive relevant degree of freedom and to build up the Landau free energy. Newer investigations [24] indicate that the high-temperature unreconstructed phase is in fact dynamically disordered so that the average structure is only effectively unreconstructed whereas the atoms jump among some equivalent positions near their unreconstructed sites. This finding does not exclude the soft phonon behaviour but implies an important damping of this mode. When covered by a sufficient amount of $\mathrm{H}$ atoms the reconstructed surfaces switches to a different structure [25]. The analogous (001) surface of Mo seemed to show a similar reconstruction [26], with the condensed soft mode slightly displaced from the border of the Brillouin zone so that its wavelength is incommensurate with the underlying lattice. The phase transition is also driven by a soft surface phonon [27]. The later $\mathrm{X}$-ray measurements have shown that this reconstruction is in fact commensurate, but with a larger period $c(7 \sqrt{2} \times \sqrt{2}) R 45^{\circ}$.

Still larger reconstruction patterns have been found on surfaces of $5 d \mathrm{fcc}$ metals: Ir, $\mathrm{Pt}$ and $\mathrm{Au}$. On the $\mathrm{Au}(111)$ the surface atoms occupy the fcc and hcp sites with a complex pattern of $[(22 \pm 1) \times \sqrt{3}]$ which results from the tendency of the surface layer to form as much as possible compact structure [28]. The same tendency makes the (001) $\mathrm{Au}$ and (001) $\mathrm{Pt}$ reconstruct to a distorted incommensurate structure of the unit cell $(26 \times 68)$. Below $970 \mathrm{~K}$ the hexagonal pattern on $\mathrm{Au}(001)$ is slightly rotated with respect to the high-symmetry directions of the unreconstructed surface. In the range $970 \mathrm{~K}<T<1170 \mathrm{~K}$ the rotation does not exist any more, and above $1170 \mathrm{~K}$ the surface deconstructs towards a disordered state of an average structure of the unreconstructed $A u(001)$ [29]. First principle 
total energy calculations show that the tendency to the close packing of the surface is characteristic of the $5 d$ metals and not of the $4 d$ metals such as $\mathrm{Ag}$ [30].

The (110) surfaces of fcc metals $\mathrm{Au}$ and $\mathrm{Pt}$ show the $(2 \times 1)$ missing row reconstruction. The slopes of the resulting valleys are again portions of the closely packed structure. In $1170 \mathrm{~K}$ the reconstructed surface of $\mathrm{Au}$ deconstructs to a flat disordered phase whereas the corresponding surface of $\mathrm{Pt}$ deconstructs at $1820 \mathrm{~K}$ to a rough phase [31].

\subsection{Surfaces of semiconductors}

The elemental.semiconductors $\mathrm{Si}$ and $\mathrm{Ge}$ are characterised by strong valence bonds. The cleavage of surfaces makes some of them dangle freely. The reconstruction of their surfaces is, therefore, related to formation of new bonds on the surface. The $\mathrm{Si}(111)$ was investigated for a long time and the final statement of its structure has been given by STM measurements. The surface is reconstructed in a $(7 \times 7)$ pattern involving dimers, adatoms, and stacking faults (DAS). The clean surface deconstructs at $1110 \mathrm{~K}$ to a disordered $(1 \times 1)$ one [32]. Upon deposition of $\mathrm{Ag}$ atoms in $240 \mathrm{~K}$, the $(7 \times 7)$ structure transforms to a $(\sqrt{3} \times \sqrt{3})$ one [33]. A number of other reconstructed surfaces of semiconductors are known [34].

\subsection{Surfaces of minerals}

The reconstruction of other types of surfaces is not so well known as for metals and semiconductors. The (001) surface of rutile $\mathrm{TiO}_{2}$ has shown to be reconstructed by $(1 \times 3)$ by an STM observation [35]. The polar surfaces of ruby $\alpha-\mathrm{Al}_{2} \mathrm{O}_{3}$ which has the corundum structure is reconstructed so that it remains an epitaxial deposed layer of the spinel structure $\gamma-\mathrm{Al}_{2} \mathrm{O}_{3}[36]$.

\section{Rough surfaces}

The best evidence of rough surfaces exists for fcc metals. Their close packed surfaces (111) and the surfaces (001) do not show any roughening. The (110) surfaces show, on the contrary, a variety of reconstruction and roughening phenomena. The unreconstructed $\operatorname{In}(110), \mathrm{Pb}(110), \mathrm{Cu}(110)$ and $\mathrm{Ni}(110)$ roughen in $T_{\mathrm{R}}=290 \mathrm{~K}, 390 \mathrm{~K}, 1100 \mathrm{~K}$ and $1310 \mathrm{~K}$ respectively [37]. The reconstructed $\mathrm{Pt}(110)$ roughens at $1080 \mathrm{~K}$, whereas the $\mathrm{Au}(110)$ first deconstructs to a disordered flat phase which subsequently roughens about $50 \mathrm{~K}$ above, i.e. at about $750 \mathrm{~K}$. Recent reviews on the subject $[31,38]$ discuss an interesting interplay between reconstruction and roughening of these surfaces. A roughening has been also observed for molecular crystals: biphenyl, naphtalene and tetrabromomethane [2].

\section{References}

[1] J.C. Heyraud, J.J. Métois, J. Cryst. Growth 50, 571 (1980); Surf. Sci. 128, 585 (1964); A. Pavlovska, K. Faulian, E. Bauer, Surf. Sci. 221, 233 (1989); A. Pavlovska, M. Tikhov, Y. Gu, Surf. Sci. 278, 303 (1992).

[2] A. Pavlovska, D. Nenow, Surf. Sci. 27, 211 (1971); J. Cryst. Growth 12, 9 (1972); ibid. 39, 346 (1977). 
[3] J.C. Heyraud, J.J. Métois, J. Cryst. Growth 8, 269 (1987); A. Pavlovska, D. Dobrev, E. Bauer, Surf. Sci. 326, 101 (1995).

[4] H.J. Ernst, E. Hulpke, J.P. Toennies, Phys. Rev. Lett. 52, 1941 (1987).

[5] N. Takeuchi, C.T. Chan, K.M. Ho, Phys. Rev. B 43, 13899 (1991).

[6] M.S. Daw, M.I. Baskes, Phys. Rev. B 29, 6443 (1984).

[7] F. Ercolesi, E. Tosatti, M. Parinello, Phys. Rev. Lett. 57, 719 (1986).

[8] Yu.E. Kitaev, I.P. Ipatova, Prog. Surf. Sci. 18, 189 (1985); D.M. Hatch, H.T. Stokes, Phase Transit. 7, 87 (1986); K. Osuch, Phase Transit. 12, 147 (1988).

[9] V. Chirita, B.A. Pailthorpe, Thin Solid Films 208, 149 (1992); J. Mościński, W. Alda, M. Bargiel, Acta Phys. Pol. A 80, 649 (1991).

[10] S.K. Ma, Statistical Mechanics, World Scientific, Philadelphia 1985, p. 488.

[11] M. den Nijs, Phys. Rev. B 40, 4709 (1989).

[12] K. van Beijeren, Phys. Rev. Lett. 38, 993 (1977).

[13] G. Brudeylins, R.B. Doak, J.P. Toennies, Phys. Rev. Lett. 46, 437 (1981); G. Benedek, G. Brudeylins, R.B. Doak, J.G. Skofronick, J.P. Toennies, Phys. Rev. $B$ 27, 3662 (1983); Phys. Rev. B 28, 2104 (1983).

[14] F.W. de Wette, W. Kress, U. Schröder, Phys. Rev. B 32, 4143 (1985).

[15] N. Bickel, G. Schmidt, K. Heinz, K. Müller, Phys. Rev. Lett, 62, 2009 (1989).

[16] J. Prade, U. Schröder, W. Kress, J. Phys., Condens. Matter 5, 1 (1992).

[17] P. Zieliniski, K.H. Michel, Phys. Rev. B 46, 4815 (1992).

[18] B. Piveteau, D. Spanjaard, M.D. Desjonquers, Phys. Rev. B 49, 8402 (1994); A.M. Rodriguez, G. Bozzolo, J. Ferrante, Surf. Sci. 289, 100 (1993).

[19] F. Jona, P.M. Marcus, in: The Structure of Surfaces II, Eds. J.F. van der Veen, M.A. Van Hove, Springer, Berlin 1988, p. 90.

[20] H.L. Davis, J.B. Hannon, K.B. Ray, E.W. Plummer, Phys. Rev. Lett. 68, 2632 (1992); P.T. Sprunger, K. Pohl, H.L. Davies, E.W. Plummer, Surf. Sci. 297, L48 (1993).

[21] M.K. Debe, D.A. King, J. Phys. C 10, L303 (1977); T.E. Felter, R.A. Barker, P.J. Estrup, Phys. Rev. Lett. 38, 1138 (1977).

[22] C.L. Fu, A.J. Freeman, E. Wimmer, Phys. Rev. Lett. 54, 2261 (1985).

[23] A. Fasolino, E. Tosatti, Phys. Rev. B 35, 4264 (1987).

[24] W. K. Han, S.C. Ying, Phys. Rev. B 48, 14524 (1993).

[25] K. Kankaala, T. Ala-Nissula, S. Ch. Ying, Phys. Rev. B 47, 2333 (1993).

[26] A. Fasolino, E. Tosatti, Phys. Rev. B 35, 4264 (1987).

[27] G.-C. Wang, T.M. Lu, Surf. Sci. 122, L635 (1982).

[28] N. Takeuchi, C.T. Chan, K.M. Ho, Phys. Rev. B 43, 13899 (1991).

[29] D. Gibbs, B.M. Ocko, D.M. Zehner, S.G.J. Mochrie, Phys. Rev. B 42, 7330 (1990).

[30] N. Takeuchi, C.T. Chan, K.M. Ho, Phys. Rev. B 43, 14363 (1991).

[31] J. Lapujoulade, Surf. Sci. Rep. 20, 191 (1994).

[32] E. Bauer, in: Structure and Dynamics of Surfaces II, Eds. W. Schommers, P. von Blanckenhagen, Springer, Berlin 1987, p. 115; J. Boland, Surf. Sci. 244, 1 (1991). 
[33] A. Shibata, Y. Kimura, K. Takayanaga, Surf. Sci. 303, 161 (1994).

[34] R. Feidenhans'l, Surf. Sci. Rep. 10, 105 (1989).

[35] P.W. Murray, F.M. Leibsle, C. A. Muryn, H.J. Fisher, C.R.J. Flipse, G. Thornton, Phys. Rev. Lett. 72, 689 (1994).

[36] L.A. Bursill, P.J. Lin, Philos. Mag. 60, 307 (1989).

[37] J.C. Heyraud, J.J. Métois, J. Cryst. Growth 8, 269 (1987); P. Zeppenfeld, K. Kern, R. David, G. Comsa, Phys. Rev. Lett.62, 63 (1989); Y.C. Cao, E.H. Conrad, Phys. Rev. Lett. 64, 447 (1990).

[38] M. Bernasconi, E. Tosatti, Surf. Sci. Rep. 17, 363 (1993). 